\title{
Amantadine Resistant of Indonesian H5N1 Subtype Influenza Viruses
} During 2003-2008

NI LUH PUTU INDI DHARMAYANTI ${ }^{1 *}$, FERA IBRAHIM ${ }^{2}$ AND AMIN SOEBANDRIO ${ }^{2,3,4}$

'Virology Department, Balai Besar Penelitian Veteriner, Jalan RE Martadinata 30, Bogor 16114, Indonesia; 'Microbiology Department, Universitas Indonesia, Jalan Pegangsaan Timur 16, Jakarta 10320, Indonesia;
${ }^{3}$ State Ministry of Research and Technology, Gedung II BPP Teknologi Jalan MH Thamrin 8, Jakarta 10340, Indonesia; 'Indonesia National Committee for Avian Influenza Control and Pandemic Influenza Preparedness.

The M2 protein of 146 avian influenza (AI) viruses data available in public database (NCBI), including $20 \mathrm{AI}$ isolates used in this study were sequenced at the M2 protein to find out the probability of mutation and the increase of resistance to amantadine after more than 5 years of
their circulation in Indonesia. The results showed that during 2003-2008, around $62.58 \%$ (92/147) AI viruses in Indonesia have showed resistance to amantadine and 10 of them have dual mutations at V27 Aand S3 $1 \mathrm{~N}$.

Key words: amantadine, resistant, $\mathrm{H} 5 \mathrm{~N} 1$ subtype influenza virus

In Indonesia, the H5N1 Influenza disease has circulated for more than 5 years, since its outbreak in 2003. In 2003, Dharmayanti et al. (2004) and Wiyono et al. (2004) for the first time identified the avian influenza (AI) H5N1 subtype Un Ar viruses still cause serious problems and have become endemic disease in poultry farms as well as

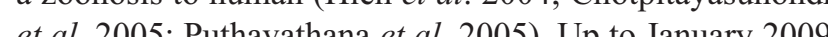

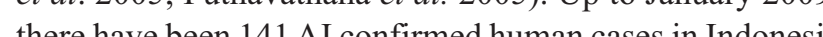
115 of the 141 a fal (WHO 2009). Thes ind

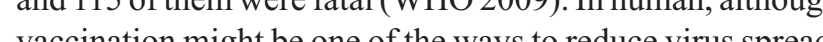
vaccination migh be one (Whe ways to reduce vins spread, vaccine preparation and its produch require more tha There are two and cing no

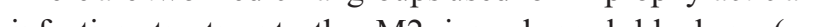
infection treant the $\mathrm{M} 2$ ion cha amantadhe and is derivatives) and the NA inibitors (e.g. (. (rimantadine 1993) Thi vis Wh 1993). The gus of mutation and is ineffectiveness for influenza B viru (Hayden and Hay 1992)

Li cist (2004) stated that most viruses isolated from South East Asta were resistant to amantadine an of antivirl drugs for the treat of in inhibiting the vinu repliction by restrining the ion chense formed by 22 pretin The s. 30 . aids (at position 26, 27,30,31 and 34) in the

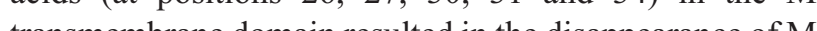

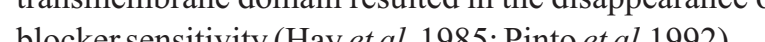

Ilyushina et al. (2005) reported about influenza A viruses that were potentials to be pandemic during 1979-1983, namely $\mathrm{H} 5, \mathrm{H} 6, \mathrm{H} 7$ and $\mathrm{H} 9$, They were detected to be nonresistant to amantadine. However, between 2000-2004 resistances to amantadine were detected in South East Asia, amounted to $31.1 \%$ for subtype $\mathrm{H} 5$ and $10.6 \%$ for subtype H9 respectively.

ore Cheung et al.(2006) in their research on the genetic mutation distribution of resistance to adamantane derivatives isolates from Vietnam, Thailand, Cambodia, Indonesia, Hong Kong and China showed that more than 95\% of isolates from Vietnam and Thailand mutated at M2 resulted in their resistance to adamantane. In Indonesia the figure was about $6.3 \%$ (2 out of 32 viruses), while in China $8.9 \%$. Generally, the mutation occurred at Leu26IleSer31Asn in almost all isolates from Vietnam, Thailand and Cambodia. In Indonesia, Smith et al. (2006) reported that the viruses from Sumatra showed mutation of Ser31Asn on M2 protein, indicating that they become resistance to amantadine. Hurt et al. (2007) stated that about 30\% (2 out of 6 (from a total of 2005) showed resistance to adamantane. Based on these studies, the increase of genetical virus diversities, and the frequent human and animal influenza
outbreaks in Indonesia, the present study was focused on finding the possibilities of mutations at M2 protein in 2003 2008 It is hoped that new information on virus resistance to amantadine in Indonesia would be obtained.

\section{MATERIALS AND METHODS}

AI Virus. The 20 viruses used were isolated in 20032008 (Table 1) and identified as subtype H5N1 avian influenza virus (Dharmayanti et al. 2004; 2005a, b, c; 2006; 2008). They were propagated in 9-11 days old embryonated

specific pathogen free (SPF) eggs.
RT-PCR-DNA Sequencing. The extraction of RNA viruses was conducted using QIAmp viral RNA mini kit with a slight modification. The full length Matrix gene amplification was con Thed by one step RT-PCR system with RT-PCR that had been optimized by Dhitrogen) (2009). The Matrix primer used was the one followed by Hoffman et al. (2001).

The amplified DNA was purified using QIAquick gel purification kit (Qiagen). The sequencing method used was 
Table 1 Avian influenza H5N1 subtype viruses collected from different AI cases

\begin{tabular}{|c|c|c|}
\hline No & Viruses & $\begin{array}{ll}\text { Sample origin } \\
\end{array}$ \\
\hline $\begin{array}{l}1 \\
2\end{array}$ & $\begin{array}{l}\text { A/Ck/West Java/1074/2003 } \\
\text { A/Ck/East Java/BL-IPA/2003 }\end{array}$ & Outbreak in the layer chicken farm without AI vaccination \\
\hline 3 & A/Muscovyduck/Jakarta/DKI-Uwit/2004 & \\
\hline & A/Duck/Banten/Pdgl-Kas/2004 & \\
\hline 5 & A/Ck/Jakarta/DKI31/2005 & AI outbreak in the backyard poultry \\
\hline 6 & A/Muscovyduck/Bgr-Cw/2005 & \\
\hline 7 & A/Ck/West Java/Smi-Hay/2005 & \\
\hline 8 & A/Muscovyduck/Jakarta/DKI-Sum106/2006 & The viruses were collected from AI backyard poultry \\
\hline & A/Duck/Jakarta/DKI-SImt306/2006 & at the surrounding $\mathrm{H} 5 \mathrm{~N} 1$ human case collected \\
\hline 10 & A/Ck/Jakarta/DKI-Nurs/2007 & surrounding $\mathrm{H} 5 \mathrm{~N} 1$ human case \\
\hline$\frac{11}{12}$ & 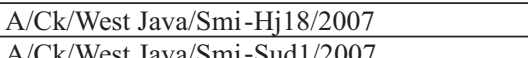 & Outbreak in the layer chicken farm with AI vaccination \\
\hline 12 & A/Ck/West Java/Smi-Sud1/2007 & Layer chicken farm with AI vaccination (no clinical sign) \\
\hline 13 & A/Muscovyduck/West Java/Bks3/2007 & The viruses were collected from $\mathrm{AI}$ outbreak in the \\
\hline $\begin{array}{l}14 \\
15\end{array}$ & $\begin{array}{l}\text { A/Ck/Pessel/BPPVRII/2007 } \\
\text { A//k/Lhu/BPPVRI/2007 }\end{array}$ & backyard poultry at the surrounding $\mathrm{H} 5 \mathrm{~N} 1$ human case \\
\hline 16 & A/Ck/West Java/Smi-Acul/2008 & Al outbreak in the backvard poultry \\
\hline$\frac{17}{18}$ & $\mathrm{~A} / \mathrm{Ck} / \mathrm{Banten} / \mathrm{Srg}-\mathrm{Fadh} / 2008$ & \\
\hline & A/Ck/West Java/Smi-M1/2008 & O \\
\hline 19 & A/Ck/West Java/Smi-M6/2008 & Outbreak in the layer chicken farm with AI vaccination \\
\hline
\end{tabular}

direct sequencing using Cycle sequencing kit (BigDye Terminator version 3.1; Applied Biosystem). The nucleotid sequencing data obtained were analyzed simultaneously with the M2 gene sequencing data available from avia influenza database. The multiple sequence alignments was conducted using Clustal W program (www.ebi.ac.uk), protein translation and data manipulation were carried out using Bioedit program, whereas the phylogenetic tree was reated using MEGA 4 program (www.megasoftware.net).

Amantadine Sensitivity Assay. The resistant test on amantadine was carried out by using cell-based virus reduction assay using the method developed by Cheung $e t a l$. (2006) with minor modification. MDCK was grown up to $90 \%$ confluent in a 12 hole plate (Nunc), infected with $30 \mu \mathrm{L}$ virus $\left(10^{\circ} \mathrm{EID}_{50}\right)$ with the presence of amantadine hydrochloride (sigma) $0.1,1,4$ and $8 \mu \mathrm{g} \mathrm{mL}^{-1}$. Each treatment was in three replicates. MDCK and virus that had been treated with amantadine were incubated for 3-4 days. The supernatant in the plate holes were individually tested for the

\section{RESULTS}

The results of M2 protein sequencing and translation from the 20 isolates were 97 amino acids. It was found tha $62.58 \%$ or $92 \mathrm{AI}$ viruses in Indonesia are resistant to amantadine. The substitution of a single amino acid at sites 26 (Leu $\rightarrow$ Phe), 27 (Val $\rightarrow$ Ala or Thr), 30 (Ala $\rightarrow$ Thr or Val), 31 (Ser $\rightarrow$ Asn or $\mathrm{Arg}$ ) and $34(\mathrm{G} \rightarrow \mathrm{E})$ in the transmembrane domain of M2 resulted in the missing of M2 blocking sensitivity causing resistance to amantadine (Hay et al. 1985; Pinto et al. 1992; Suzuki et al. 2003). Fifty eight out of the 92 mutants had the mutation at position 27 (Val $\rightarrow$ Ala/Thr; V27A), 24 viruses had it at position 31 $(\mathrm{Ser} \rightarrow \mathrm{Asn} / \mathrm{Arg}$; S31N), while 10 viruses showed dual

Twenty isolates were tested in vitro for the resistance to amantadine at MDCK cells. The results of HA tests showed that there was a consistent correlation between resistant and sensitive viruses at the molecular levels. For instance, the 2003-2005 viruses (numbers 1-6) were sensitive to amantadine, showed also sensitivity absence of virus titer (virus titer $=0$ ) inhibition by the lowe $(0.1 \mathrm{\mu g} \mathrm{mL}-1)$ amantadine concentration. By exposing amantadine resistant viruses (numbers 7-20) to higher of amantine $\left(8 \mathrm{H} \mathrm{mL}^{-1}\right)$ they remained

The phylogenetic tree analysis of M2 gene showed that the influenza viruses from Indonesia are in a different group from the Hong Kong and China ones (Fig 1). The 7 viruses from the avian species outbreaks showed its close relationship to other viruses of NCBI avian species-origina data. The others demonstrated genetic proximity at Matrix gene level with human-origin viruses. The 5 viruses showed dual mutations, namely the $\mathrm{A} / \mathrm{Ck} / \mathrm{WJ} / \mathrm{Smi}-\mathrm{Hj} 18 / 2007$, $\mathrm{A} / \mathrm{Ck} / \mathrm{WJ} / \mathrm{Smi}-\mathrm{sud} 1 / 2007, \mathrm{~A} / \mathrm{Ck} / \mathrm{WJ} / \mathrm{Smi}$-Biot/2008, $\mathrm{A} / \mathrm{Ck} / \mathrm{WJ} / \mathrm{Smi}-\mathrm{M} 1 / 2008$, and $\mathrm{A} / \mathrm{Ck} / \mathrm{WJ} / \mathrm{Smi}-\mathrm{M} 6 / 2008$ They were isolated from intensively AI vaccinated commercial poultry farms and have proximities to the source of A/Indonesia/CDC1047/2007 which was also showed duat mutations, i.e. V27A and $\mathrm{S} 31 \mathrm{~N}$. The remaining 2 viruses, amely the $\mathrm{A} / \mathrm{Ck} / \mathrm{WJ} / \mathrm{Smi}-\mathrm{Acul} / 2008$ and the $\mathrm{A} / \mathrm{Ck} / \mathrm{B}$ ten $/ \mathrm{Srg}-\mathrm{Cadh} / 2008$ from an AI non-human chicken outbreak, showed a single mutation at V27A.

\section{DISCUSSION}

The mRNA of M2 protein is transcribed from the segment 7 of RNA descended from the coliner of M transcript by splicing. M2 is a protein that has a membran spanning domain that also provides a signal for the transportation to the cell surface. The presence of a large number of tetramers on the surface of infected cells and ew in virions is believed to have the role of ion proto channel controlled by the golgi pH during the HA synthesi

\begin{tabular}{|c|c|c|c|c|c|c|c|c|c|}
\hline \multirow[t]{2}{*}{0} & \multirow{2}{*}{$\begin{array}{l}\text { NCBI } \\
\text { Acc No } \\
\end{array}$} & \multirow[t]{2}{*}{ Viruses } & \multicolumn{2}{|l|}{ Amino acid posisition } & \multirow[t]{2}{*}{ No } & \multirow{2}{*}{$\begin{array}{l}\text { NCBB } \\
\text { Acc No }\end{array}$} & \multirow[t]{2}{*}{ Viruses } & \multicolumn{2}{|l|}{ Amino acid posisition } \\
\hline & & & $\begin{array}{llll}26 & 27 & 30 & 31 \\
\end{array}$ & & & & & $\begin{array}{llll}26 & 27 & 30 & 31 \\
\end{array}$ & \\
\hline & 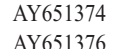 & 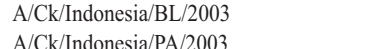 & $\begin{array}{ll}\mathrm{L} & \mathrm{V} \\
\mathrm{L} & \mathrm{V}\end{array}$ & & $\begin{array}{l}75 \\
76\end{array}$ & $\begin{array}{l}\text { CY0144306 } \\
\text { CY014314 }\end{array}$ & $\begin{array}{l}\text { A/IIdonesiaiCDC599/20006 } \\
\text { A/ndonesialCDC53/2006 }\end{array}$ & & \\
\hline & 51377 & 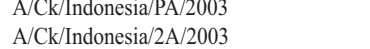 & $\begin{array}{lll}\mathrm{L} & \mathrm{V} \\
\mathrm{L} & \mathrm{V} & \end{array}$ & $\mathrm{G}_{\mathrm{G}}^{\mathrm{a}}$ & ${ }_{77}^{10}$ & $\begin{array}{l}\text { CY1014414 } \\
\text { CY01369 }\end{array}$ & 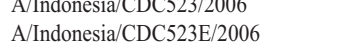 & $\begin{array}{lll}\mathrm{L} & \mathrm{A} & \mathrm{A} \\
\mathrm{L} & \mathrm{A} & \mathrm{A}\end{array}$ & \\
\hline & DQ320995 & Achicken/Wonosoboo/BPPV4/2003 & A & $\mathrm{G}$ & 78 & CY014385 & Alndonesia/ CDC58222006 & $\mathrm{L}$ & \\
\hline & DQ492906 & Achicken Pekalongan BP & $\mathrm{v}$ & $\mathrm{G}$ & 79 & CY014397. & A/Indonesia/CDC6101/2006 & $\mathrm{L}$ & \\
\hline & DQ492907 & A/chicken/Sragen/BPPV4/2003 & A S & G & 80 & $\begin{array}{l}\text { CY014405 } \\
C Y 01413\end{array}$ & A/Indonesia/CDC623/2006 & $\mathrm{L}$ & \\
\hline & EF473084 & $\begin{array}{l}\text { A/chicken/Indonesia/1/1// } \\
\text { ACk West alava } 107420\end{array}$ & $\begin{array}{llll}\text { L } & \text { V } & \text { S } & \text { S } \\
\text { L } & \text { V } & \text { A } & \text { S }\end{array}$ & $\begin{array}{l}G \\
G\end{array}$ & $\begin{array}{l}81 \\
82\end{array}$ & $\begin{array}{l}\text { CY014413 } \\
\text { CY01421 }\end{array}$ & $\begin{array}{l}\text { A/Indonesia/CDC623E/2006 } \\
\text { A/ndonesialCC624/2006 }\end{array}$ & $\mathrm{L}_{\mathrm{L}}$ & \\
\hline & & $\begin{array}{l}\text { A/k/West Java/10/4/2003 } \\
\text { A/Ck/West Java/BL-IPA/2003 }\end{array}$ & $\begin{array}{llll}\text { L } & \text { V } & \text { A } & \text { S } \\
\text { L } & \text { V } & \text { A } & \text { S }\end{array}$ & & $\begin{array}{l}82 \\
83\end{array}$ & $\begin{array}{l}\text { CY014429 } \\
\text { CY01429 }\end{array}$ & $\begin{array}{l}\text { A/ninonessald LC6424/2000 } \\
\text { A//ndonesia/CDC624E/2006 }\end{array}$ & $\begin{array}{l}\mathrm{L} \\
\mathrm{L}\end{array}$ & \\
\hline & & $\begin{array}{l}\text { A/Dk/Indonesia/MS } / 2004 \\
\text { A }\end{array}$ & ${ }_{\mathrm{A}}^{\mathrm{A}}$ & & & CY014437 & A/Indonesia/CDC625/2006 & $\begin{array}{l}\mathrm{L} \\
\mathrm{L}\end{array}$ & \\
\hline & AY651378 & A/CK/Indonesia/4/2004 & & & & CY014445 & Alndonesia/CDC6342006 & $\mathrm{L}$ & \\
\hline & AY651379 & A/CKIIndonesia/5/2004 & $\begin{array}{llll}\mathrm{L} & \mathrm{V} & \mathrm{A} & \mathrm{S} \\
\mathrm{V}\end{array}$ & G & 86 & CY014453 & A/Indonesia/CDC634P/2006 & A & \\
\hline & & 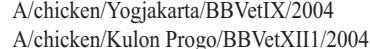 & $\begin{array}{llll}\text { L } & \text { V } & \text { A } & \text { S } \\
\text { L } & \text { V } & \text { A } & \text { S }\end{array}$ & & $\begin{array}{l}87 \\
88\end{array}$ & $\begin{array}{l}\text { CY0141461 } \\
\text { CY014469 }\end{array}$ & $\begin{array}{l}\text { A/ndonesialCCD633TT/2006 } \\
\text { AlndonesialCDC599N/2006 }\end{array}$ & $\mathrm{L}$ & \\
\hline & & $\begin{array}{l}\text { Achrcken Kunon Progo/ } \\
\text { Achicken/Malang BBVe }\end{array}$ & $\mathrm{v}$ & & ${ }_{89}^{88}$ & $\begin{array}{l}\text { Yrov } 44699 \\
\text { CY01478 }\end{array}$ & 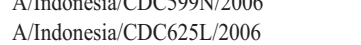 & $\begin{array}{l}\mathrm{L} \\
\mathrm{L}\end{array}$ & \\
\hline & & 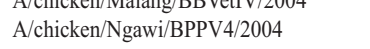 & A & & 90 & CY014485 & A/Indonesia/CDC699/2006 & $\begin{array}{l}\mathrm{L} \\
\mathrm{L}\end{array}$ & \\
\hline & DQ492908 & A/quail/BoyolaliliBPVV4 & $\mathrm{v}$ & G & 91 & CY014493 & A/Indonesia/CDC669P/2006 & & \\
\hline & $\begin{array}{l}\text { DQ4929210 } \\
\text { Do492913 }\end{array}$ & A/quail/Yogiakarta/BB & $\begin{array}{ll}\mathrm{V} & \mathrm{A} \\
\mathrm{V} & \mathrm{A}\end{array}$ & G & 92 & CY014501 & A/Indonesia/CDC669/2006 & $\mathrm{L}$ & \\
\hline & $\begin{array}{l}\text { DQ4992913 } \\
\text { DQ492914 }\end{array}$ & 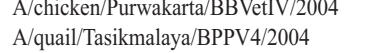 & 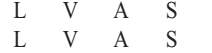 & $\begin{array}{l}\mathrm{G} \\
\mathrm{G}\end{array}$ & 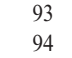 & $\begin{array}{l}\text { CY01414506 } \\
\text { CY014514 }\end{array}$ & 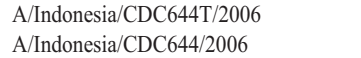 & ${ }_{\mathrm{L}}^{\mathrm{L}}$ & \\
\hline & DQ492915 & A/chicken/B & $\begin{array}{lll}\text { L } & \text { V } & A \\
\end{array}$ & G & 95 & CY014377 & A/Indonesia/CDC523T/2006 & $\mathrm{L}$ & \\
\hline 22 & $\begin{array}{l}\text { DQ4492916 } \\
\text { DQ492917 }\end{array}$ & $\begin{array}{l}\text { Achicken'Bangli BaliliBB } \\
\text { Achicken JJembrana/BPP }\end{array}$ & 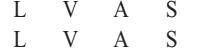 & $\begin{array}{l}\mathrm{G} \\
\mathrm{G}\end{array}$ & $\begin{array}{l}96 \\
97\end{array}$ & $\begin{array}{l}{ }_{\text {CY01717641 }} \\
\text { CY01749 }\end{array}$ & 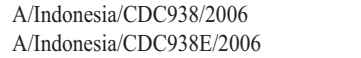 & $\begin{array}{ll}\mathrm{L} & \mathrm{A} \\
\mathrm{L} & \mathrm{A}\end{array}$ & \\
\hline & DQ492918 & Acchicken MangarainTT/BP & $\mathrm{V} \quad \mathrm{A}$ & G & 98 & CY017657 & A/Indonesia/CDC940/2006 & $\mathrm{L}$ & \\
\hline & $\begin{array}{l}\text { DQ4492921 } \\
\text { DQ492922 }\end{array}$ & 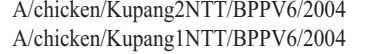 & $\begin{array}{llll}\mathrm{L} & \mathrm{V} & \mathrm{A} & \mathrm{S} \\
\mathrm{L} & \mathrm{V} & \mathrm{A} & \mathrm{S}\end{array}$ & $\begin{array}{l}G \\
G\end{array}$ & $\begin{array}{r}99 \\
100\end{array}$ & $\begin{array}{l}\text { YY0017655 } \\
\text { CY017773 }\end{array}$ & 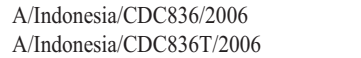 & $\begin{array}{ll}\mathrm{L} & \mathrm{A} \\
\mathrm{L} & \mathrm{A}\end{array}$ & \\
\hline & DQ492923 & A/chicken/Pangkalpinang/BP & $\begin{array}{lll}\mathrm{L} & \mathrm{V} & \mathrm{A} \\
\mathrm{C}\end{array}$ & G & 101 & $\begin{array}{l}\text { CY017681 } \\
C Y 1420\end{array}$ & $\begin{array}{l}\text { A Indnonesia/ CDC8353/2006 } \\
\text { A }\end{array}$ & $\mathrm{L}$ & \\
\hline & & $\begin{array}{l}\text { AtturkeyKed } \\
\text { AchickenKu }\end{array}$ & $\begin{array}{llll}\mathrm{L} & \mathrm{V} & \mathrm{A} & \mathrm{S} \\
\mathrm{L} & \mathrm{V} & \mathrm{A} & \mathrm{S}\end{array}$ & $\begin{array}{l}G \\
G\end{array}$ & $\begin{array}{l}102 \\
103\end{array}$ & $\begin{array}{l}{ }_{\text {CY01414532 }} \\
\text { CY0446 }\end{array}$ & $\begin{array}{l}\text { A/IIndonesia//CDCC33/92006 } \\
\text { Alndonsesia/CDC75/2006 }\end{array}$ & $\begin{array}{ll}\mathrm{L} & \mathrm{A} \\
\mathrm{L} & \mathrm{A}\end{array}$ & \\
\hline & GU183429 & AMuscovyd & $\begin{array}{llll}\mathrm{L} & \mathrm{V} & \mathrm{A} & \mathrm{S} \\
\mathrm{S}\end{array}$ & & 104 & 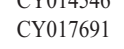 & $\begin{array}{l}\text { Ambonts } \\
\text { Alndonsia CDC88772006 }\end{array}$ & $\begin{array}{lll}\mathrm{L} & \mathrm{A} \\
\mathrm{A} & & -1\end{array}$ & \\
\hline & $\begin{array}{l}\text { GUU183430 } \\
\text { DO320994 }\end{array}$ & 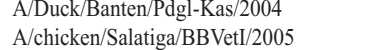 & ${ }^{\mathrm{A}}$ & $\mathrm{G}$ & 105 & EU146818 & AIndonesialas83H/2006 & $\mathrm{L}$ & \\
\hline & & 5 & $\begin{array}{lll}\mathrm{V} & \mathrm{A} \\
\mathrm{A} & \mathrm{A}\end{array}$ & G & $\begin{array}{l}106 \\
107\end{array}$ & 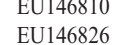 & 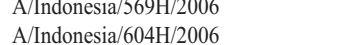 & $\begin{array}{l}\mathrm{L} \\
\mathrm{L}\end{array}$ & \\
\hline & & & & & & & 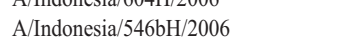 & & \\
\hline & & chicker & A & & & EU146787 & Alndonesial $560 \mathrm{H} / 2006$ & $\mathrm{v}$ & \\
\hline & & A/chicken/P & A & G & & EU146778 & AIndonesia/542H/2006 & $\mathrm{L}$ & \\
\hline & & & $\begin{array}{ll}\mathrm{V} & \mathrm{A} \\
\mathrm{V} & \mathrm{A}\end{array}$ & & & & A/ndonesia/546H/2006 & $\mathrm{V}$ & \\
\hline & & 列 & $\begin{array}{ll}\mathrm{V} & \mathrm{A} \\
\mathrm{V} & \mathrm{A} \\
\mathrm{A}\end{array}$ & G & 1112 & 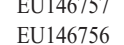 & 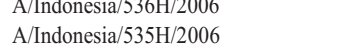 & $\begin{array}{l}\mathrm{L} \\
\mathrm{L}\end{array}$ & \\
\hline & & & $\begin{array}{r}\mathrm{V} \\
\mathrm{C}\end{array}$ & $\mathrm{G}$ & & & 12006 & $\begin{array}{l}\mathrm{L} \\
\mathrm{L}\end{array}$ & \\
\hline & & & & & & $\mathrm{EU} 146802$ & Alndonesia/567H/2006 & $\mathrm{L}$ & \\
\hline & & A/chich & A & & 116 & & & $\mathrm{~L}$ & \\
\hline & & 5 & $\begin{array}{l}\mathrm{A} \\
\mathrm{A}\end{array}$ & G & 117 & EUI. & A/Indonesia/341H/2006 & $\mathrm{L}$ & \\
\hline & & (B) & $\begin{array}{lll}V & A \\
V & A\end{array}$ & G & $\begin{array}{l}118 \\
119\end{array}$ & & $\begin{array}{l}\text { Allndones } \\
\text { Alndones }\end{array}$ & $\mathrm{L}$ & \\
\hline & & & $\begin{array}{rll}\mathrm{V} & \mathrm{A} \\
\mathrm{A}\end{array}$ & & 120 & & 2006 & $\begin{array}{l}\text { A } \\
\text { A }\end{array}$ & \\
\hline & & & A & $\mathrm{G}$ & 121 & & Alndon & $\begin{array}{l}\mathrm{A} \\
\mathrm{V}\end{array}$ & \\
\hline & CY01 & Achicken!! & A & & 122 & $\mathrm{EU} 146690$ & Alndonesia/286H/2006 & $\mathrm{L}$ & \\
\hline & & Allndones & A & G & 123 & EU146683 & Allndonesial2835 & L A & \\
\hline & $\begin{array}{ll}C Y 0 \\
C Y 0\end{array}$ & $\begin{array}{l}\text { Alldonon } \\
\text { Alndone }\end{array}$ & $\begin{array}{l}\text { A } \\
\text { A }\end{array}$ & G & $\begin{array}{l}124 \\
125\end{array}$ & & $\begin{array}{l}\text { Sum106/2006 } \\
\text { S2006 }\end{array}$ & $\mathrm{L}$ & \\
\hline & & & $\begin{array}{ll}A & A \\
A & A\end{array}$ & $\mathrm{G}$ & & & & L & \\
\hline & & & A A & & & & & $\begin{array}{l}\mathrm{L} \\
\mathrm{L}\end{array}$ & \\
\hline & & Indonesi: & A & G & & & & A & \\
\hline & & AIndones & A & & & & 2/2007 & A & \\
\hline & & & v & & 130 & CY019387 & A/l & A & \\
\hline & & & A & & & & & A & \\
\hline & & & $\begin{array}{lll}\mathrm{V} & \mathrm{A} \\
\end{array}$ & $\mathrm{G}$ & & & & A & \\
\hline & & & $\begin{array}{lll}\mathrm{V} & \mathrm{A}\end{array}$ & $\mathrm{G}^{-}$ & & & & $A_{A}$ & \\
\hline & & & A & & & & & $\begin{array}{l}\mathrm{A} \\
\mathrm{A}\end{array}$ & \\
\hline & & & & & & & & 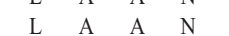 & \\
\hline & & & & & & & $\mathrm{KI}-\mathrm{H}$ & A & \\
\hline & & & & G & & & & A & \\
\hline & & & & & & & & A & \\
\hline & & & & $\mathrm{G}$ & & & 些ar $B$ & ${ }^{A}$ & \\
\hline & & & & & & & 2007 & $\begin{array}{l}\mathrm{A} \\
\mathrm{A}\end{array}$ & \\
\hline & & & & & & & & $\mathrm{A}^{\mathrm{A}}$ & \\
\hline & & & & & & & & $\mathrm{A}$ & \\
\hline & & & & & & & & & \\
\hline & & & & & & & & & \\
\hline & & & & & & & & & \\
\hline
\end{tabular}




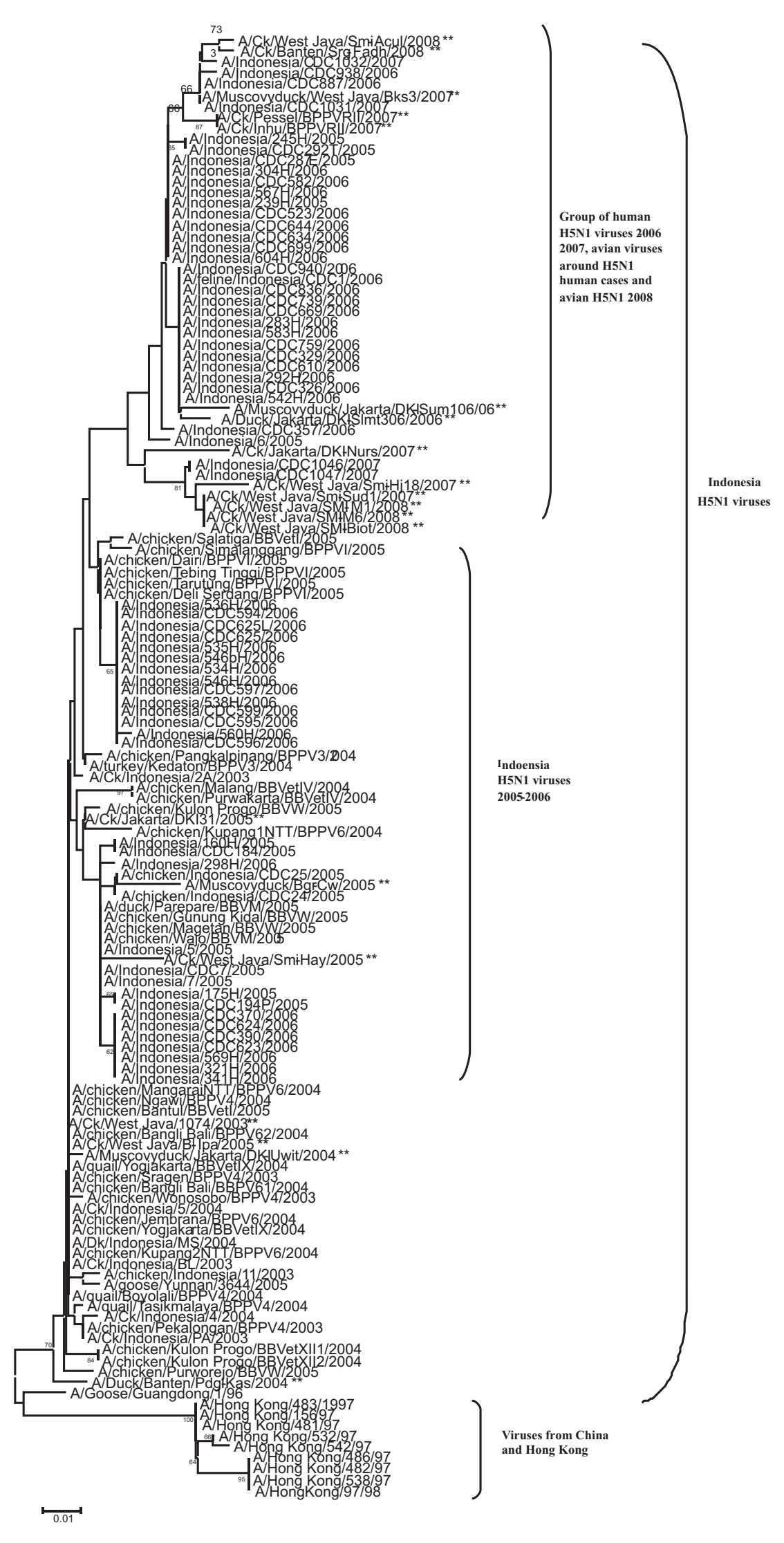

Figure 1 Phylogenetic tree of Indonesia M2 H5N1 subtype influenza viruses. The viruses used in this study is marked by double star character. followed by the interior acidification of virions while uncoating viruses (Webster et al. 1992).

In previous study, Bright et al. $(2005,2006)$ stated that although the resistant to amantadine $\mathrm{H} 5 \mathrm{~N} 1$ virus is presence in Asia, most of its spread is in Vietnam and Thailand. Most of the $\mathrm{H} 5 \mathrm{~N} 1$ viruses in Indonesia and China are still sensitive to amantadine. Most of the influenza viruses $(70-80 \%)$ showed the mutation at position 31 of the M2 protein and around $1-2 \%$ at position 26 . Meanwhile mutations at two locations, namely Leu26Ile and Ser31Asn, are very (1 out of the 1307 available publications of sequence database for influenza A virus, i.e. the $\mathrm{A} / \mathrm{Swine} / \mathrm{Scotland} / 410440 / 94$ (Marozin et al. 2002). Cheung et al. (2006) stated that the high mutation occurrence on Leu26lle and specifically its relation with Ser31Asn only occurred in H5N1 viruses isolated from Vietnam, Thailand, and Cambodia, indicated that dual mutations is due to a selection pressure as there was no single mutation of Leu26lle or Ser31Asn among the ant viruses

Those studies showed that Indonesian viruses are to amantadine and only a few mutated. (92) the Ind dition increase at M2 protein.

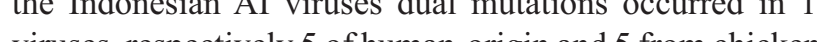
(V27 A S31N) The first do log CDC157/2006. Next, in 2007 the wect 4 visses

The present study showed that dual virus mutation were found routinely in chickens vaccinated for AI. Five viruse hreviouly, mutation previously occurred in human. Pring a single m $31 \mathrm{~N}$ S at S3IN position and the numer gradually increased an a new viruses. As shown to new vires. As shown also by Pun Indonesian viruses could not inhibit HJN1 viruses, eve All Indonesin viruses in the present sto

All Indonesian viruses in the present study showed the same mulation pattern, namis at positions 27 and 31 (V27 and S3IN),; No of the muted assitions 26 and 34. This is quite differt for whe the muted in Vetnam, Thailand and Cambodia, where the mutation generally reveled that the Noth Vitan. Later Le da (2008) 2007 wa replaced by coude 23.4 , that were 1 HN 1 in 2007 was replaced by clade 2.3.4, hat were sensitive to anate combination of anartit suggested.

Results from the in vitro of amantadine resistant test showed no difference in capability against increase viru titers, both by using single (V27A or S3IN) or dual (V27A and S3IN) mutations. It seens

\section{REFERENCES}

Bright RA, Marie-jo M, Xu X, Perez-Oronoz G, Wallis TR, Davis XM, Povinelli L, Nancy J Cox NJ, Klimov AI. 2005. Incidance of
adamantine resistance among influenza A (H3N2) viruses isolated worldwide from 1994 to 2005 : a cause for concern. Lancet 366:1175-

Bright RA, Shay DK, Shu B, Cox NJ, Klimov AI. 2006. Adamantane resistance among influenza A viruses isolated early during the $1995-206$ influenza season in the United States. JAMA 295:891-4.
200 . Chotpitayasunondh T, Ungehusak K, Hanshaoworakul W, Chunsuthiwat S,
Sawanpanyalert P. 2005. Human disease from influenza A (H5N1), Thailand. 2004. Emerging Infect Dis 11:201-9.

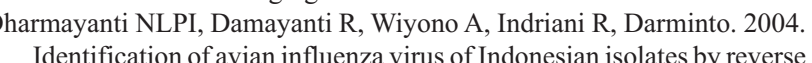
trantification of avian influenza virus of Indonesian isolates by reverse
transcriptase polymerase chain reaction (RT-PCR) method. JITV 9:136-43.

Dharmayanti NLPI, Damayanti R, Indriani R, Wiyono A, Adjid RMA. 2005a. Molecular characterization of Indonesia avian influenza virus.

2005b. Indonesian avian influenzza viruses character in second wave the outbreaks. JITV 10:217-26.

armayanti NLPI, Indriani R, Adjid RMA. 2006. Identification and Ragunan. MKH 22:79-83.

Dharmayanti NLPI, Indriani R, Damayanti R, Wiyono A. 2005c. Isolation and identification of avian influenza outbreak in October 2004 - March

Dharmayanti NLPI, Indriani R, Hartawan R, Hewajuli DA, Ratnawati A,
Darminto. 2008. Genetic mapping of Indonesian avian influenza viruses in 2007. J Biol Indon 5:155-71.

Cheung CL, Rayner RM, Smith GJD, Wang P, Naipospos TSP, Zhang J, Distribution of amantadine-resistant H5N1 Avian Influenza variants in Asia. J Infect Dis 193:1626-9.

Hay AJ, Wolstenholme AJ, Skehel JJ, Smith MH. 1985. The molecular basis of the specific anti-influenza action of amantadine. EMBO $J$

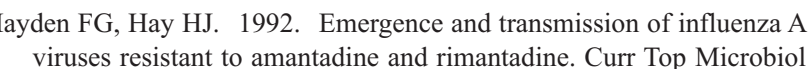
Immunuol 176:119-30.

Hien TT, de Jong M, Farrar J. 2004. Avian influenza: a challenge to global ,

priner set for the full-lenth amplification of DR. 2001. Universal Arch Virol 146:2275-89. Susceplow 2007. the neuramidase inhibitors and adamantanes. 2007. Antiviral llyushina NA, Govorkova EA, Webster RG. 2005. Detection of amantadine-resistant variant among avian influenza viruses isolated in Le MTQ, Wertheim HFL, Nguyen HD, Taylor W, Hoang PVM, Vuong CD,
Nguyen HLK, Nguyen HH, Nguyen TQ, Nguyen TV, Van TDcek!!, Ngoc BT, Bui TN, Nguyen BG, Nguyen LT, Luong ST, Phan PH, Pham HV, Nguyen T, Fox A, Nguyen CV, Do HQ, Crusat M, Farrar J,
Nguyen HT, de Jong MD, Horb P. 2008. Influenza A H5N1 Clade 2.3. Virus with a different antiviral susceptibility profile Virus in humans in Northern Vietnam. Plos One 10:33339:1-8, doi 10.137.

Li K.S, Guan Y, Wang J, Smith GJD, Xu KM, Duan L, Rahardjo AP,
Puthavathana PBuranthai C, Nguren TD, Estoepangestie ATS, Chaisingh A, Auewarakul P Long HT, Hanh NTH, Webby RI, Poon
Cont LLM, Chen H, Shortridge KF, Yuen KY, Webster RG, Peiris JSM. 2004. Genesis of a highlypathogenic and potentially pandemic $\mathrm{H} 5 \mathrm{~N} 1$

Influenza virus in eastern Asia. Nature 430:209-13.
Marozin S, Gregory V, Cameron K, Bennett M, Valette M, Aymard M, Foni
E, Barigzzi G, Lin Y, among swine influenza HIN1 and N1N2 viruses in Europe. J Gen Virol 83:735-45. 
Smith GJD, Naipospos TSP, Nguyen TD, de Jong MD, Vijaykrishna D, Usman TB, Hassan SS, Nguyen TV, Dao V, Bui NA, Leung YHC, Cheung CL, Rayner JM, Zhang JX, Zhang LJ, Poon LLM, Li KS, Nguyen VC, Hien TT, Farrar J Webster RG, Chen H, Peiris SSM, Guan
Y. 2006. Evolution and adaptation of $\mathrm{H} N \mathrm{N1}$ influenza virus in avian and human hosts in Indonesia and Vietnam. Virology 350: 258-68. Suzuki H, Saito R, Masuda H, Oshitani H, Saito M, Sato I. 2003
Emergence of amantadine-resitstant influenza A viruses: epidemiological study. J Infect Chemother 9:195-200.

C RA. 1992. Influenza virus M2 protein has ion channel activity. Cell 69:517-28.
Puthavathana P, Auewarakul P, Charoenying PC, Sangsiriwut K, Pooruk P, Boonnak K, Khanyok R, Thawachsupa P, Kijphati R, Sawanpanyalert P. 2005. Molecular characterization of the complete genome of human influenza H5N1 virus isolates from Thailand. J Gen Virol 86:423-33. Isolasi dan karakterisssi virus highly pathogenis Aminto. 2004. subtipe H5 dari ayam asal Wabah di indonessia. JTTV 9:61-71. Wang C, Takeuchi K, Pinto LH, Lamb RA. 1993. Ion channel activity of influenza A virus M2 protein: characterization of amantadine block. J (c)

-ecology ofinfluenza A virun OT, Kawaoka Y. 1992. Evolution and 